\title{
La resurrezione dei morti nel De principiis di Origene: note di confronto con alcuni testi precedenti
}

\author{
Alberto D'Anna \\ UNIVERSITÀ "ROMA TRE" \\ DIPARTIMENTO DI STUDI UMANISTICI \\ alberto.danna@uniroma3.it
}

Il tema della resurrezione dei morti in Origene è stato studiato approfonditamente da alcuni dei migliori esperti dell'opera e del pensiero dell'Alessandrino ${ }^{1}$. In tempi recenti, Emanuela Prinzivalli ha proposto, a più riprese, un'analisi dettagliata e una mirabile sintesi dell'argomento ${ }^{2}$. Sarebbe dunque fuori luogo, oltre che presuntuoso, tentare da parte mia di riproporre una visione complessiva del pensiero origeniano in merito a questo articolo di fede; un pensiero che, com'è noto, ha raggiunto un grado di complessità elevatissimo ed è stato al centro -insieme con le tesi a esso connesse circa la successione dei mondi, l'apocatastasi, l'assenza della carne, la salvezza del Diavolo- di feroci e durature polemiche. Più semplicemente, tenterò qui di percorrere alcuni brani del De principiis e di proporre qualche confronto con altri testi precedenti, attinenti alla stessa materia. Ne risulteranno, credo, sia le peculiari caratteristiche dottrinali ed esegetiche della riflessione dell'Alessandrino, sia anche alcuni punti di contatto -talvolta inaspettati- con le fonti considerate.

1 Cf., p.e., H. Crouzel, «La doctrine origénienne du corps ressuscité», in Bulletin de littérature ecclésiastique 81 (1980), 175-200; G. DORIVAL, «Origène et la résurrection de la chair», in L. Lies (ed.), Origeniana Quarta (Innsbrucker theologische Studien, 19; Tyrolia-Verlag, Innsbruck-Wien 1987), 291-321.

2 E. Prinzivalli, «Resurrezione» in: A. Monaci (dir.), Origene. Dizionario (Città Nuova, Roma 2000), 401-405; EAD., «Polemiche escatologiche fra origenisti e antiorigenisti», in: EAD., Magister Ecclesiae. Il dibattito su Origene fra III e IV secolo (Studia Ephemeridis Augustinianum, 82; Institutum Patristicum Augustinianum, Roma 2002), 65-136, spec. 65-70 e 87-104; EAD., "La risurrezione nei Padri», in: S. A. Panimolle (ed.), Morte-Risurrezione nei Padri (Dizionario di Spiritualità Biblico-Patristica 45; Borla, Roma 2007), 169-288 (Origene: 210-211; 217-227). 
Nella prefazione del De principiis, la fede nella resurrezione dei morti è inclusa nel novero delle "verità che in maniera chiara sono state tramandate dalla predicazione apostolica" (I, praef., 4) e vi è sinteticamente descritta più con parole di Paolo che dello stesso Origene: "E ci sarà il tempo della resurrezione dei morti, quando questo nostro corpo, che ora è seminato nella corruzione, risorgerà nella incorruttibilità e, seminato nell'ignominia, risorgerà nella gloria [1Cor 15, 42-43]" (I, praef., 5)3. Vedremo presto che questa modalità di enunciazione, originale rispetto a quella di tutti gli altri articoli di fede presentati in praef., 4-104, prelude alla centralità dell'esegesi di Prima ai Corinzi 15 nella trattazione per esteso del tema, nonché all'insistenza sul cambiamento di condizione dei risorti, che caratterizzano la riflessione origeniana.

In De principiis II, 9 Origene ricapitola la trattazione sulle creature razionali, parlando in ultimo (Princ. II, 9, 5-8) della giustizia dell'unico Dio; da essa dipendono sia l'ordine dell'eone presente, sia i castighi futuri (che sono comunque medicinali) oppure la futura retribuzione dei meriti, dei quali "il computo preciso [...] conosce solo Dio con la sua parola e la sua sapienza unigenita e il suo Spirito santo" (Princ. II, 9, 8).

Ciò lo porta ad affrontare i temi del giudizio e della retribuzione. "Ma per arrivarci ordinatamente" -aggiunge- "mi sembra opportuno parlare prima della resurrezione, per sapere che cosa sarà ciò che andrà al supplizio o al riposo e alla felicità". La resurrezione, dunque, è introdotta in De principiis II, 10, 1 come premessa universale degli eventi ultimi (sempre da considerare, comunque, in senso relativo, nella prospettiva origeniana dell'apocatastasi).

L'universalità della resurrezione, anche altrove chiaramente affermata da Origene ${ }^{5}$, non era, ancora alla sua epoca, un dato unanimemente

3 Le traduzioni italiane dei passi del De principiis sono tratte da: ORIGENE, I Principi, a cura di M. Simonetti (Classici delle religioni. La religione cattolica; U.T.E.T., Torino 1968).

4 In tali paragrafi, quella di 1Cor 15, 42-43 è visibilmente la citazione scritturistica più significativa, perché è la più ampia e soprattutto perché è essa stessa a descrivere il contenuto dell'articolo di fede; negli altri casi, invece (I, praef., 4: Gv 1, 3, Fil 2, 7; I, praef., 8: $\operatorname{Rm} 7,14,1 \operatorname{Cor} 12,8$ ) le citazioni o le allusioni sono brevi e addotte a rinforzo dell'esposizione del tema fatta da Origene con parole proprie.

5 Cf. Comm. in Rom. V, 9: "Poiché poi l'apostolo dice in altri passi che noi siamo ormai risorti con Cristo [...] mentre qui lo dice come cosa che attualmente è futura e da sperare, questo è il motivo per cui si pensa a una duplice resurrezione: una, 
condiviso. Nelle fonti cristiane dei primi secoli, così come in quelle giudaiche coeve o di poco anteriori, si rintracciano sia la concezione della resurrezione universale, presupposto necessario alla retribuzione (Daniele; Secondo libro dei Maccabei; Atti degli Apostoli; Vangelo di Giovanni $\left.{ }^{6}\right)$, sia quella della resurrezione identificata con la retribuzione "positiva" e, dunque, riservata solamente ai salvati; una concezione, quest' ultima, che presuppone anche la considerazione negativa di una forma di sopravvivenza priva della componente corporea dell'uomo (Primo libro di Enoch; Salmi di Salomone; Mishnah; Talmud Babilonese; Didachè; Lettera di Clemente ai Corinzi; Ignazio; Terza lettera ai Corinzi; Lettera di Barnaba; i cristiani criticati da $\left.\mathrm{Celso}^{7}\right)^{8}$. La persistenza nel terzo secolo di questa concezione di per sé retributiva della resurrezione la si può dedurre, $a$ contrario, dalle puntualizzazioni esegetiche di Ippolito, Cipriano e Lattanzio" sull'interpretazione di Salmo 1, 5 -"gli empi non risorgeranno in giudizio"- miranti a spiegare il versetto non come escludente i malvagi

secondo cui con la mente, l'intenzione e la fede risorgiamo con Cristo dalle realtà terrene per meditare sulle realtà celesti e ricercare quelle future; l'altra, che sarà una resurrezione generale di tutti nella carne. Pertanto, quella resurrezione che è secondo la mente, in base alla fede, sembra già realizzata in coloro che pensano alle cose che sono lassù dove sta Cristo alla destra di Dio $[\mathrm{Col} 3,1]$. Invece la resurrezione generale della carne che riguarda tutti è ancora futura: quella infatti si è compiuta nella prima venuta del Signore, questa si compirà nella seconda”. Per quanto riguarda l'uso, in questo brano, della formula "resurrezione della carne", se anche essa non sia ascrivibile alla versione di Rufino, occorre ricordare che, occasionalmente, Origene se ne serve, riferendosi con tale espressione "dei semplici" al sostrato materiale amorfo, impermanente e mutevole: cf. E. Prinzivalli, «Polemiche escatologiche fra origenisti e antiorigenisti», 99, n. 137.

6 Dn 12, 2-3; 2Mac 7, 14; At 24, 15; Gv 5, 28-29.

7 IEnoch 46, 6; 48, 10; Salmi di Salomone 3, 10-12; Mishnah, Sanhedrin 11; Talmud Babilonese, Sanhedrin 90; Didachè 16, 5; Lettera di Clemente ai Corinzi 26; IGNAZIO, Agli Smirnesi 2; Terza lettera ai Corinzi 2, 24-25; Lettera di Barnaba 11, 7; 15, 7; 21, 1; C. Cels. V, 14.

8 Sulle fonti giudaiche, cf. N. T. Wright, Risurrezione (Pensiero Cristiano. Strumenti 28; trad. it. Claudiana, Torino 2006; orig. inglese SPCK, London 2003), 201; 233-243; su quelle cristiane cf. E. Prinzivalli, "La risurrezione nei Padri», 176178; 215. Quanto a Paolo, fonte privilegiata da Origene nella trattazione del tema della resurrezione, nelle lettere autentiche non esprime una posizione dottrinale ben definita, poiché parla solo della resurrezione di "quelli di Cristo" (cf. 1Ts 4, 16; 1 Cor 15, 23), ma non nega esplicitamente quella dei peccatori e parla diffusamente di un giudizio universale (cf., p.e., $R m$ 2, 1-11).

9 Ippolito, Bened. Isaac. PO 27, 155; Cipriano, Test. ad Quir. III, 31; LatTanzio, Div. Inst. VII, 20. 
dalla resurrezione, ma come prova che essi sono già stati giudicati. Ed è lo stesso Origene che, altrove, proprio nel suo commento al Salmo 1, parzialmente tramandatoci da Metodio e da Epifanio ${ }^{10}$, afferma che "i fedeli più semplici ritengono che gli empi non saranno partecipi della resurrezione e non saranno ritenuti degni del giudizio divino".

I paragrafi II, 10, 1-2 sono rivolti contro i negatori della resurrezione del corpo: dovrebbe trattarsi di gnostici (valentiniani e basilidiani) e marcioniti, spesso accostati tra loro da Origene ${ }^{11}$, anche se l'espressione "praecipue haeretici" potrebbe indicare che sono considerati anche i sostenitori ecclesiastici della resurrezione della sola anima, sui quali si tornerà tra breve.

Tutti costoro ammettono la resurrectio mortuorum. Origene inizia ad argomentare soffermandosi sul secondo membro del sintagma, il complemento di specificazione "dei morti". Se la resurrezione è propriamente di ciò che è morto, non può essere dell'anima, che Dio ha voluto immortale, almeno dal punto di vista fisico (si veda anche Contro Celso V, 24: "siamo perfettamente d'accordo [con Celso] che [Dio] può concedere la vita eterna dell'anima, e non solo può, ma anche la concede") ${ }^{12}$; di necessità, dunque, oggetto della resurrezione dev'essere il corpo, che muore. L'affermazione iniziale di Origene richiama, sotto certi aspetti, l'inizio del terzo frammento del De resurrectione dello Pseudo-Giustino: "La resurrezione è della parte carnale morta, perché lo spirito non muore"13. È interessante notare che in questo discorso apologetico, prodotto certamente in un ambiente di cultura asiana (lato sensu, secondo la concezione proposta da Manlio Simonetti) e per altri risguardi assai lontano dalla posizione origeniana (basti pensare al termine oळ@xíov che definisce ciò che risorge, anche se subito appresso l'autore utilizza

10 Metodio di Olimpo, De resurrectione I, 20-24; Epifanio di Salamina, Panarion 64, 10-12.

11 Cf. Princ. II, 7, 1; 9, 5; e i passi citati da M. Simonetti in Origene, I Principi, 297, n. 9.

12 Cf. E. Dal Covolo, «Note sulla dottrina origeniana della morte», in R. Daly (ed.), Origeniana Quinta. Papers of the 5th International Origen Congress (Boston College, 14-18 August 1989) (Bibliotheca Ephemeridum Theologicarum Lovaniensium, 105; Leuven University Press - Peeters, Leuven 1992), 430-437.

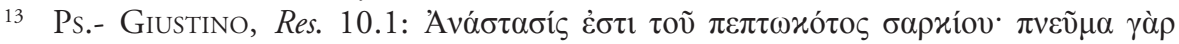

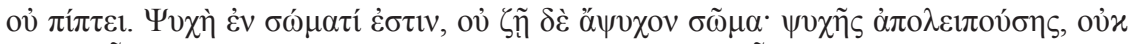

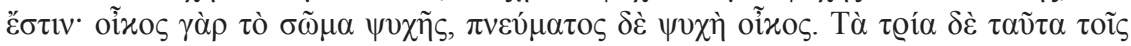

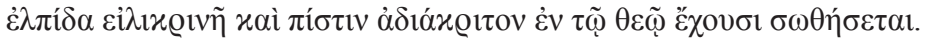


per tre volte $\sigma \tilde{\omega} \mu \alpha)$, si coglie l'esito, analogo a quello del De principiis, di un percorso che gradualmente porta al restringimento semantico della confessione della "resurrezione dei morti": da restituzione dell'intero uomo alla vita (così in Paolo) a rivivificazione di ciò che è morto. Premessa di una tale evoluzione semantica della confessione di fede è, non solo in ambiente alessandrino ma anche in quello asiano (cf. Giustino, Ireneo ${ }^{14}$ ), l'approfondimento della riflessione antropologica e il convincimento che l'anima, pur se creata, per volontà di Dio non muore (contro l'equazione generazione $=$ corruzione $)$.

Il riferimento scritturistico subito introdotto da Origene ("è seminato un corpo animale, risorge un corpo spirituale") è tratto dal quindicesimo capitolo della Prima lettera ai Corinzi (qui 15, 44), il testo principale, come si è detto, che Origene utilizza a proposito della resurrezione e, più in generale, della concezione escatologica. Di fatto, quel capitolo costituisce la più ampia e articolata trattazione sulla resurrezione presente nei testi divenuti autoritativi per i cristiani e lo troviamo, infatti, al centro dell'aspetto esegetico del dibattito e delle polemiche intorno a quel punto nodale della fede professata. Dovremo tornarci. Per adesso mi limito a notare che il versetto citato funge da cardine, nella breve sezione rivolta a gnostici e marcioniti, per passare dal primo asserto sull'oggetto della resurrezione -il corpo- al secondo asserto, sulle qualità del corpo spirituale risorto -incorruttibilità e immortalità- e per prevenire in tal modo la possibile obiezione di una morte successiva alla resurrezione, data la presenza del corpo. Il ragionamento di Origene si conferma, così, essere orientato qui alla considerazione del solo eone presente, visto che, invece, nella prospettiva di una successione di mondi, non sarebbe in realtà da escludere proprio una successione anche di morti e resurrezioni del corpo della stessa creatura razionale che non abbia progredito (o regredito) dalla condizione umana ${ }^{15}$.

Un'ulteriore annotazione mi sembra necessaria, prima di procedere oltre: Origene attribuisce il rinnovamento del corpo alla gratia et virtus resurrectionis. Vale a dire che, sia pure con un breve accenno, egli distingue tra il discorso sulle modalità della resurrezione e sulle diverse qualità di corpo morto e corpo risorto e il discorso sulla causa di tale trasfor-

14 Giustino, Dial. 6, 1-2; Ireneo, Adv. Haer. II, 34, 4; V, 1, 3; 6, 1; 7, 1; 12, 1-3; Dem. 11.

15 Cf. E. Prinzivalli, «La risurrezione nei Padri», 226. 
mazione; questa risiede, in ultima istanza, nella volontà divina, come

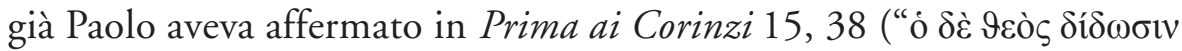

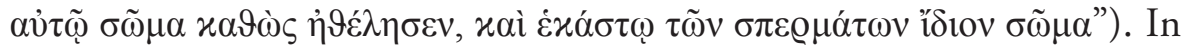
tale breve accenno è comunque implicita un'importante presa di distanza dalla concezione naturalistica della resurrezione, che affiora qua e là in fonti cristiane anteriori (Lettera di Clemente ai Corinzi; Teofilo d'Antiochia $^{16}$ ) e che riconduce di fatto la resurrezione ad un necessarismo ciclico. Non a caso, trattando della resurrezione nel Contro Celso, Origene polemizza, questa volta sul fronte filosofico, con il ritorno degli stessi individui postulato dalla cosmologia stoica ${ }^{17}$. La libertà dell'azione divina in relazione alla resurrezione sarà poi ribadita più ampiamente $\mathrm{da}$ Origene in De principiis III, 6, $5^{18}$.

Il paragrafo II, 10, 3 segna il cambiamento degli interlocutori cui Origene rivolge la sua attenzione e nei confronti dei quali intende sviluppare la sua riflessione: "alcuni dei nostri che, per pochezza d'intelligenza e scarsezza di spiegazioni, hanno concezione molto bassa e volgare della resurrezione del corpo".

Ricordando di nuovo le parole dell'Apostolo sulla trasformazione del corpo risorto (1Cor 15, 42-44), come argomento autorevole usato in maniera preventiva, questa volta nei confronti dei nuovi interlocutori, Origene li accusa di contraddire tale auctoritas prospettando un corpo ancora soggetto alle passioni: "è fuor di luogo e contro il suo [di Paolo] pensiero dire che il corpo sarà ancora soggetto alle passioni della carne e del sangue". Una tale accusa, in realtà, fonde insieme due concezioni sulla resurrezione che troviamo, a volte, in effetti congiunte, altre volte, invece, nettamente separate: si tratta del ritorno, alla resurrezione,

\footnotetext{
Lettera di Clemente ai Corinzi 24-25; Teofilo D'Antiochia, Ad Aut. I, 13.

17 C. Cels. V, 23: "Noi non diciamo, dunque, che il corpo, dopo essersi corrotto, ritorna alla sua natura originaria. [...] I filosofi stoici, invero, sostengono che il corpo completamente putrefatto ritorna alla sua natura primitiva, poiché essi sostengono il principio dell'assoluta identità dei cicli del mondo".

18 Il successivo sintetico discorso sulla forma dei corpi risorti (Princ. II, 10, 2), qui enunciato soprattutto in chiave polemica, è comprensibile, com'è ben noto, alla

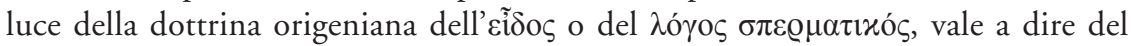
principio che garantisce la continuità tra corpo terreno e corpo risorto di ciascun individuo. Essa è accennata anche poco oltre (II, 11,3), ma è sviluppata soprattutto in C. Cels. IV, 56-57 e nel commento al Salmo 1, in Epifanio, Panarion 64, 1415. Su questa tesi origeniana si veda E. Prinzivalli, «Polemiche escatologiche fra origenisti e antiorigenisti», 96-100.
} 
del corpo materiale dell'uomo e del ritorno, con esso, anche delle sue funzioni, comprese quelle alimentari e sessuali, nelle quali Origene non fatica a individuare la causa di alcune passioni ${ }^{19}$.

È interessante rilevare i punti di contatto -negli argomenti usati e nella procedura dialettica- tra la polemica condotta qui dall'Alessandrino e quella dei negatori ecclesiastici della salus carnis, (sebbene questi siano forse tra gli obiettivi -come s'è detto- della polemica precedente: Princ. II, 10, 1-2), dei quali ci dà testimonianza il De resurrectione dello Pseudo-Giustino e che Antonio Orbe ha chiamato "origenisti ante Origenem" 20 . Tali cristiani asserivano che, se la carne risorge, "chiaramente avrà anche gli organi genitali $[\tau \grave{\alpha} \mu$ ó@ı $\alpha$ : ma non sarebbe assurdo affermare che questi esisteranno dopo la resurrezione dai morti, dal momento che il Salvatore ha detto: «Non prenderanno moglie né marito, ma saranno come angeli nel cielo»? Gli angeli [...] non hanno carne né mangiano né hanno unioni sessuali; pertanto neppure ci sarà una

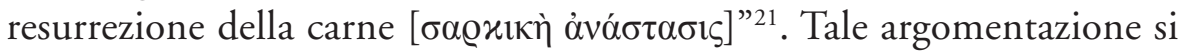
fondava sulla combinazione, in un sillogismo ipotetico del secondo tipo stoico, di un postulato medico-filosofico e di un detto autorevole di Cristo: il primo (lasciato in realtà sottinteso) è quello, tipico della fisiologia

19 Non a caso sono quello alimentare e quello sessuale i primi due impulsi naturali menzionati più avanti, in Princ. III, 2, 2, come cause del peccato concorrenti con gli impulsi demoniaci.

20 Il grande studioso ha distinto un gruppo di avversari di Ireneo dagli gnostici e dai marcioniti: A. Orbe, "Adversarios anónimos de la Salus Carnis», in Gregorianum 60 (1979), 9-53. Appartenevano alla "grande chiesa", negavano la salvezza della carne e intendevano la resurrezione come ascensione della sola anima a un luogo sopraceleste, al momento della morte, e a Dio, alla fine del mondo. Erano spinti a disprezzare il mondo materiale e a svalutare il corpo rispetto all'anima dalla buona formazione filosofica, in particolare platonica. Praticavano un'ampia allegoresi della Scrittura. Nel monumentale commentario al libro V dell'Adversus haereses, Orbe sostiene che quegli avversari anonimi erano gli stessi affrontati dall'autore del $D e$ resurrectione: A. Orbe, Teología de San Ireneo. Comentario al Libro V del "Adversus haereses», I-III (BAC maior 25; 29; 33; La Editorial Católica, Madrid-Toledo 19851988; per rintracciare le note sui negatori ecclesiastici della salvezza della carne, si veda l'Indice di eretici e dottrine eterodosse, vol. III, 794-795, sotto la voce "Dimidia salus"). Ho discusso e cercato di sviluppare la tesi di Orbe in: Pseudo-Giustino, Sulla resurrezione. Discorso cristiano del II secolo, a cura di A. D'AnNA (Letteratura cristiana antica. Testi; Morcelliana, Brescia 2001), 243; 254-277.

21 Ps.-Giustino, De res. 2, 3. 
di Galeno ${ }^{22}$, della consequenzialità necessaria tra organo e funzione, tra

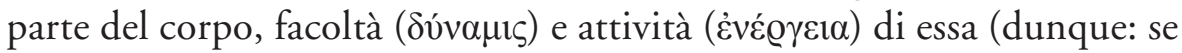
risorge la carne, ritornano le sue funzioni); le parole di Cristo ${ }^{23}$, smentendo l'apodosi della prima premessa, ne avrebbero contraddetto anche la protasi, come gli stessi utilizzatori di questo argomento esplicitamente mostravano.

La stessa necessaria consequenzialità tra presenza degli organi e delle loro funzioni appare sottintesa dalla frase di Origene. La confutazione dell'Alessandrino, però, presenta un'analogia solo parziale nella procedura dialettica seguita. Anch'egli, infatti, per smentire l'idea che, alla resurrezione, ritorni la carne e, con essa, i desideri che provoca, ricorre ad un asserto scritturistico autorevole, in questo caso al celeberrimo versetto di 1Cor 15, 50 ("carne e sangue non possono ereditare il regno di Dio”), introdotto immediatamente dopo il riferimento alle passioni; su tale citazione si dovrà subito tornare. Tuttavia Origene, citando Paolo, intende andare alla radice della questione e smentire direttamente la possibilità che il corpo risorto possa essere caratterizzato da carne e sangue. Anch'egli, cioè, mostra la correlazione tra la presenza di un corpo carnale e delle passioni, già di per sé ostacolo all'idea di un ritorno della carne; ma poi non si attarda in una confutazione propriamente sillogistica, ma introduce un argumentum ex auctoritate che impedisce, a suo avviso, in maniera univoca e definitiva di immaginare, nella resurrezio-

22 Per Galeno, nel corpo non vi è nulla di inutile né di inattivo, ma tutto è predisposto nel migliore dei modi e secondo una precisa e specifica finalità dalla natura ordinatrice, cioè dal Demiurgo (cf., p.e., GALENo, Sulle facoltà naturali I, 6 Helmreich; Sull'utilità delle parti IV, 2; XVI, 2; XVII, 1 Helmreich). La fisiologia galenica si radica in quella, teleologica, di Aristotele, ma la supera e ne accentua il finalismo, colto fin nel più infimo fenomeno biologico, secondo una sensibilità teologica medioplatonica, che lo porta a riconoscere ovunque l'effetto della legislazione provvidenziale del Demiurgo: cf. M. Vegetti in: GALEno, Opere scelte (Classici della Scienza; U.T.E.T., Torino 1978), 35-41; e I. Garofalo in: ibidem, 293-318.

23 La cui formulazione più estesa, citata in un successivo passo del De resurrectione (3 7: "I figli di questo mondo prendono moglie e marito, ma i figli del tempo futuro non prendono moglie né marito, ma saranno come angeli nel cielo") appare come un'armonia evangelica basata su Luca, con un moderato apporto degli altri Sinottici; cf. A. D'AnnA, Terza lettera ai Corinzi - Pseudo-Giustino, La risurrezione (Letture cristiane del primo millennio, 44; Paoline, Milano 2009), 275-276. È qui assente, tuttavia, l'interpretazione encratita del detto di Gesù data da Luca 20, 3436 e colta con acume da E. Prinzivalli, «La risurrezione nei Padri», 185-186. 
ne, la presenza di un corpo materiale: "caro et sanguis regnum dei non possidebunt".

È possibile che Origene abbia seguito questa linea argomentativa anche perché, come si diceva sopra, in realtà non tutti coloro che professavano la resurrezione della carne -non soltanto come formula di fede (anche a Origene capita di usarla), ma credendo nell'effettiva presenza nei risorti anche della componente materiale- accompagnavano tale concezione con quella del ritorno dei godimenti corporali, delle passioni (per dirla con l'Alessandrino). Certo, abbiamo ampia testimonianza di quanto, ancora all'epoca di Origene, fossero diffuse dottrine millenariste radicali, che immaginavano un regno terreno dei risorti, insieme con Cristo, caratterizzato da godimenti materiali: non si trattava di posizioni professate solamente da pochi eterodossi di epoche anteriori, quali ad esempio Cerinto ${ }^{24}$, ma da gruppi numerosi, "intere chiese” nelle parole di Dionigi di Alessandria, che narra, nel De promissionibus citato da Eusebio (H. E. VII, 24), del millenarismo materialista di Nepote, vescovo di Arsinoe e contemporaneo di Origene, e dei suoi seguaci, da Dionigi stesso ricondotti all'ortodossia e all'unità ecclesiale. Tuttavia, sia un millenarista esplicito come Giustino sia il suo allievo autore del De resurrectione, entrambi sostenitori della fede nella "resurrezione della carne" 25 , manifestano una valutazione severa nei confronti dell'attività sessuale già in questa vita ${ }^{26}$ e il secondo, nel suo discorso, è esplicito nell'escluderla totalmente in quella dei futuri risorti, rifiutando il rapporto di necessaria conseguenza tra presenza del corpo materiale e ritorno delle sue funzioni e ribaltando, contro i suoi avversari, lo stesso detto di Gesù da loro usato, inteso però come prova della cessazione delle funzioni e

4 Sul quale cf. Eusebio, H. E. III, 28.

25 Sull'uso della formula "resurrezione della carne" in Giustino, cf. quanto annoto in PSEUdo-Giustino, Sulla resurrezione, 96-97, n. 21.

26 Cf. Giustino, 1Apol. 15, 6-7; 29, 1 e il parallelo Ps.- Giustino, De res. 3, 4; Giustino, Dial. 23, 3 e Ps.- Giustino, De res. 3, 5. Nel caso del passo del Dialogo con Trifone, il parallelismo col De resurrectione è forte se si conserva il testo tradito dal Paris. graec. 450 ("neppure ve ne è bisogno ora [sc. della circoncisione], dopo

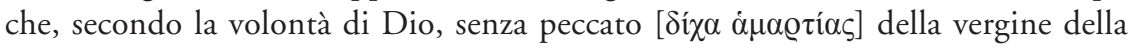
stirpe di Abramo, fu generato il Figlio di Dio Gesù Cristo") e non lo si corregge come abitualmente fanno gli editori, ultimi dei quali Marcovich e Bobichon

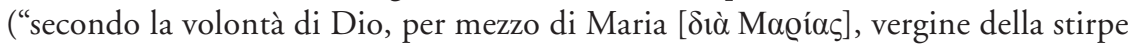
di Abramo, fu generato il Figlio di Dio Gesù Cristo”); cf. P. Prigent, Justin et l'Ancien Testament (Études bibliques, 49; Gabalda, Paris 1964), 57. 
non della mancanza di organi fisici: "[il Signore Gesù] predisse che nel mondo futuro l'unione attraverso rapporto sessuale è destinata ad essere eliminata, dal momento che dice: «I figli di questo mondo prendono moglie e marito, ma i figli del tempo futuro non prendono moglie né marito, ma saranno come angeli nel cielo»" (De res. 3, 7).

Origene, dunque, adducendo proprio il celebre versetto paolino di Prima ai Corinzi 15, 50, sbarra la strada anche alle posizioni materialiste più moderate e meditate, non soltanto a quelle estremiste e sempliciste, perché non contraddice i supposti effetti del ritorno della carne -le passioni-, ma proprio il ritorno di essa. Egli si associa, in tal modo, all'uso del versetto fatto dagli gnostici e da tutti coloro che rifiutavano l'idea che la resurrezione possa coinvolgere la componente materiale dell'uomo $\mathrm{o}$, se si preferisce - più platonicamente- il rivestimento materiale del vero soggetto umano, anima o intelletto o pneuma che sia. Ireneo, del resto, lo aveva chiaramente detto: "carne e sangue non possono ereditare il regno di Dio [...] da tutti gli eretici è addotto nella loro insensatezza, dal quale anche si sforzano di mostrare che l'opera plasmata di Dio non si salva ${ }^{27}$. Tertulliano lo aveva ribadito ${ }^{28}$.

In effetti, è stato chiaramente mostrato ${ }^{29}$ quanto tale versetto sia stato centrale nelle polemiche riguardo la fede nella resurrezione dei morti, con esiti esegetici anche paradossali. Ma prima ancora dello sfruttamento di quel versetto sul piano esegetico, a favore di una tesi o di un'altra mediante interpretazioni diverse, va registrata, come più antico tentativo a noi noto di neutralizzarne l'uso antisomatico che i primi gnostici ne facevano, la riscrittura pseudepigrafa del quindicesimo capitolo della Prima lettera ai Corinzi, costituita dalla Terza lettera ai Corinzi. Con essa, un anonimo autore dei primi decenni del secondo secolo, di area antiochena, cerca di opporsi alla concezione della resurrezione dei Saturniliani, facendo tornare a scrivere Paolo ai Corinzi e facendogli affermare in modo inequivoco che, contrariamente a quelli che dicono che "la resurrezione non è della carne" $(3 \operatorname{Cor} 1,12 ; 2,24)$, i credenti risorgeranno "avendo sana la carne" (3Cor 2, 32). Far sostenere direttamente da Paolo che il corpo materiale dell'uomo parteciperà alla resurrezione

\footnotetext{
27 IRENeO, $A d v$. har. V, 9, 1.

28 Tertulliano, De carnis resurrectione 48, 1.

29 Cf. E. Pietrella, "Caro et sanguis regnum Dei possidere non possunt" ", in Aevum 49 (1975), 36-76.
} 
mirava al duplice effetto di conferire la massima autorevolezza alla posizione dell'anonimo autore e, al contempo, di vanificare uno dei supporti più efficaci che gli avversari gnostici adducevano a favore della propria posizione. La composizione in sé della Terza ai Corinzi è, dunque, un interessante arcaico tentativo di rendere innocua l'"arma" di Prima ai Corinzi 15, 50, che gli gnostici avevano cominciato ad utilizzare; tentativo non ancora condotto sul piano esegetico, bensì mediante la pseudepigrafia apostolica, uno degli ultimi casi, da parte ecclesiastica, di ricorso a tale metodo di elaborazione e legittimazione della dottrina della fede cristiana prima dell'inizio della polemica esplicita e dell'apologetica ${ }^{30}$.

A parte, comunque, questo particolare documento, su Prima ai Corinzi 15, 50 si giocava una battaglia di tipo esegetico già prima di Origene, con inconsueti scambi di posizione rispetto ai metodi d'interpretazione, letterale o allegorico, più frequentemente utilizzati.

Come Origene, anche gli gnostici e i marcioniti -si è visto con Ireneo e Tertulliano- negavano la resurrezione della carne mediante un'esegesi strettamente letterale del versetto. In campo ecclesiastico, di Giustino (il primo dei cattolici dei quali ci è giunta un'esegesi del passo di Paolo) abbiamo conservata da Metodio di Olimpo un'interpretazione anch'essa letterale, ma tutta giocata sulla diatesi attiva e passiva del verbo "ereditare" ( $x \lambda \eta$ @ovousív), secondo la quale il corpo carnale non eredita il regno, bensì è ereditato dal regno di Dio, che è vita; dunque il corpo materiale risusciterà trasformato, senza più quelle caratteristiche di finitudine e debolezza del suo stato attuale ${ }^{31}$. Ireneo, poi, dedica un'intera

30 Un procedimento che, peraltro, sarebbe forse stato coronato da successo se la Terza ai Corinzi non fosse stata inserita, qualche decennio dopo la sua composizione, all'interno degli Atti di Paolo e con questi non avesse condiviso il rapido smascheramento e la denuncia quale opera inautentica (cf. Tertulliano, De baptismo 17, 5). Va registrato, peraltro, il suo inserimento nel più antico canone neotestamentario siriaco, la permanenza nel canone armeno fino al VII secolo, nonché le ben due versioni latine antiche e il fatto che i testimoni di esse sono manoscritti biblici. Su tutto ciò, cf. A. D'Anna, Terza lettera ai Corinzi - PseudoGiustino, La risurrezione, 24-30; 243-251.

31 Il frammento di Giustino è citato in Metodio di Olimpo, De resurrectione II, 18, 9-11: "[9] Giustino di Neapolis, uomo non lontano dagli apostoli né per tempo né per virtù, dice che è ereditato ciò che muore, mentre eredita ciò che vive, e che la carne muore, mentre vive il regno dei cieli. [10] "Quando Paolo dice

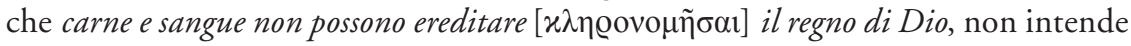

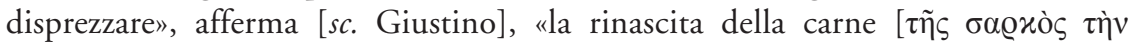


sezione del V libro dell' Adversus haereses (9, 1 - 14, 4) all'interpretazione del passo paolino; lì il maestro di Lione ripropone l'esegesi letterale di Giustino $(9,4)$, con una più esplicita menzione del ruolo dello Spirito di Dio: "colui che vive eredita i beni del morto ed altro è ereditare ed altro essere ereditato [...]. Chi è dunque colui che vive? Lo Spirito di Dio. Quali sono i beni del morto? Le membra dell'uomo che si dissolvono nella terra. Queste appunto sono ereditate dallo Spirito, in quanto sono trasferite nel regno dei cieli". A tale interpretazione, Ireneo affianca anche una di tipo morale $(11,1)$, letterale quanto al verbo, metaforica e metonimica, dunque allegorica, quanto al soggetto, ottenuta grazie alla giustapposizione di Prima ai Corinzi 15, 50 e Galati 5, 19-23: non possono prender parte al regno di Dio coloro che praticano le "opere della carne", cioè i peccati, mentre potranno ereditarlo coloro che accolgono lo Spirito e ne producono il frutto. Tertulliano, poi, propone ben quattro interpretazioni del versetto paolino: una morale, come in Ireneo ( $D e$ resurrectione carnis 49); una letterale, combinata con la precedente, che distingue tra resurrezione, propria di ogni corpo carnale (genus substantiae), ed eclusione dal regno di Dio, che non è ereditato da quella carne che si è macchiata di colpa, cioè dell'opus substantiae, e che dunque sì risorge, ma per il giudizio (De res. carnis 50, 1-3); una terza, simile alla prima di Ireneo, letterale, per cui Paolo direbbe che il corpo materiale, di per sé, non è capace del regno di Dio senza una demutatio operata dallo Spirito che dà la vita (De res. carnis 50, 4-6); infine una quarta, allegorica, secondo la quale "carne e sangue", simbolo della circoncisione, sono figura del Giudaismo, "anch'esso estraneo al regno di Dio" (De res. carnis 50, 7).

Origene, dunque, in questo caso non solo si attiene strettamente al senso letterale del testo, senza che ci risultino di lui ulteriori allegoresi del passo, ma vi si attiene secondo l'interpretazione tipica degli gnostici

$\pi \alpha \lambda \imath \gamma \gamma \varepsilon v \varepsilon \sigma i ́ \alpha v]$, ma insegnare che non il regno di Dio, che è vita eterna, è ereditato

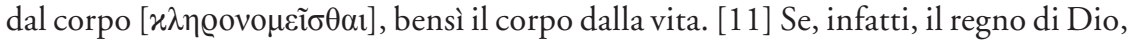
che è vita, fosse ereditato dal corpo, accadrebbe che la vita sarebbe assorbita dalla corruzione; ora, però, è la vita che eredita [ $\chi \lambda \eta \emptyset o v o \mu \varepsilon i ̃]$ ciò che è morto, affinché la morte sia assorbita in vittoria [1Cor 15, 54 ] dalla vita e ciò che è corruttibile appaia possesso dell'incorruttibilità e dell'immortalità, divenuto completamente sciolto e libero dalla morte e dal peccato, ma servo obbediente dell'immortalità, affinché il corpo sia possesso dell'incorruttibilità e non l'incorruttibilità del corpo»". Cf. P. Prigent, Justin et l'Ancien Testament, 39-41. 
e degli avversari, in genere della resurrezione della carne. Ne abbiamo conferma anche dal già citato commento al Salmo 1, dove, contro le aspettative dei simpliciores, ricorre alla stessa associazione di Prima ai Corinzi 15, 44 ("è seminato un corpo psichico, risorge un corpo spirituale") e 15, 50 che troviamo nel testo di De principiis II, 10, 3.

Il passo successivo, una volta spiegata l'impossibilità del ritorno dell'attuale corpo carnale, è quello d'introdurre il tema della trasformazione dei risorti, sempre mediante citazione di Prima ai Corinzi 15, in questo caso del versetto successivo ("tutti saremo trasformati"). Origene auspica -contro millenaristi, simpliciores, sostenitori della resurrezione della carne- che in tale trasformazione possa risiedere un'aspettativa di dignum aliquid divina gratia. Riecheggiano, in tale osservazione, analoghi passi sia del Contra Celsum, sia del commento al Salmo $1^{32}$, nei quali Origene, conformemente al principio della $\theta \varepsilon \circ \pi \varrho \varepsilon ́ \pi \varepsilon 1 \alpha^{33}$, esige che di Dio si pensino cose a lui confacenti e arriva a concordare col filosofo "nell'affermare che Dio non è il primo autore del desiderio peccaminoso, né del disordine e del traviamento, ma è solo autore della natura giusta e retta" (C. Celsum V, 24). Oggetto di una speranza degna della grazia divina è, secondo Origene, la trasformazione del corpo risorto reso per tutti comunque incorruttibile e dotato di gloria e dignità "in relazione alla dignità dell'anima e della vita di ognuno". L'Alessandrino sta accennando, dunque, alla trasformazione del sostrato materiale, lo vi $\lambda$ ıxòv

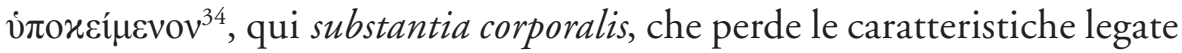
allo stato attuale della creatura razionale e si modifica secondo le nuove condizioni in cui essa si viene a trovare. Non si tratta ancora della trasformazione definitiva, corrispondente alla beatitudine finale delle anime, sulla quale -com'è noto- Origene, in De principiis II, 3 e III, 6 prospetta sia la tesi della scomparsa totale della corporeità, sia quella della corporeità sottilissima, distintiva della natura creaturale dell'anima. Comunque, proprio il carattere di stadio "intermedio" della trasformazione dovuta alla resurrezione permette di allargare questa riflessione comparativa alla parte finale del II libro, dedicata alle promesse.

\footnotetext{
Cf. Emifanio, Panarion 64, 14.

33 In proposito, cf. E. Prinzivalli, «Polemiche escatologiche fra origenisti e antiorigenisti», 97, n. 133.

34 Sul quale cf. E. PrinZIVALli, «Polemiche escatologiche fra origenisti e antiorigenisti», 98-99, n.137.
} 
La sezione di De principiis II, 11, 1-7 - de repromissionibus- richiede, infatti, almeno un breve confronto con la sezione 32-35 del V libro dell'Adversus haereses d'Ireneo, stimolato anche dal fatto che, spesso, i commentatori dell'opera di Origene indicano questo come uno dei luoghi dove più chiaramente si manifesta il contrasto tra cultura cristiana asiatica, d'impostazione materialista, e alessandrina, d'impostazione spiritualista.

Origene, in effetti, da II, 11, 2 prende nuovamente di mira coloro che "si attengono solo al significato superficiale della scrittura", "litterae solius discipuli": essi attendono "la realizzazione delle promesse nei piaceri del corpo". Sono i millenaristi già criticati in precedenza, le attese dei quali sono descritte come resurrezione della carne, vita caratterizzata dal mangiare e bere, unioni coniugali e generazione di figli, nella Gerusalemme ricostruita secondo caratteristiche mutuate tanto da Isaia quanto dall'Apocalisse; in essa saranno serviti dagli "stranieri", che pure potranno mangiare e bere, mentre i peccatori soffriranno fame e sete e saranno confusi per la vergogna. Tali millenaristi si fondano su passi dei Profeti e su altri del Nuovo Testamento "il significato dei quali non si accorgono che va inteso in senso simbolico e spirituale". Origene conclude la descrizione affermando che essi interpretano la Scrittura "Iudaico sensu".

Da parte sua, Ireneo produce un dossier scritturistico sul regno dei giusti successivo alla distruzione dell'Anticristo e al ritorno del Signore e contesta coloro che "tentano d'interpretare tali profezie in senso allegorico" $(\mathrm{V}, 35,1)$. Si tratta di "alcuni" che "sono indotti in errore dai discorsi degli eretici e ignorano le economie di Dio" (32, 1); con ogni probabilità, gli stessi avversari ecclesiastici negatori della resurrezione della carne contro i quali polemizza anche lo Pseudo-Giustino ${ }^{35}$. Si potrebbe pensare, sulle prime, che Ireneo e Origene rappresentino posizioni tra loro antitetiche.

In realtà, se esaminiamo il ricco armamentario scritturistico d'Ireneo, è facile notare che non sono molte le coincidenze con quello dei millenaristi che ha di mira Origene: il celebre detto di Gesù sul tornare a bere il frutto della vite nel regno del Padre $(M t 26,29)$, il riferimento alle beatitudini, sebbene fatto mediante due differenti macarismi ( $M t 5,5$

35 Cf. supra, n. 18. 
in Ireneo, $M t 5,6$ in Origene), alcuni versetti d'Isaia sulla ricostruzione di Gerusalemme con pietre preziose (Is 54, 11-12).

Risaltano, al contempo, altri elementi che marcano una notevole divergenza ideologica tra i due millenarismi. In Ireneo è forte la prospettiva universalista, messa in evidenza sia dalla scelta di alcune citazioni, come quella di Ezechiele 28, 25-26 o di Geremia 31, 10-14, sia direttamente ed esplicitamente dalle spiegazioni dell'autore (V, 34, 3). I millenaristi di Origene citano invece soprattutto brani di rivalsa degli Israeliti nei confronti dei gentili, dai quali sembra delinearsi una triplice divisione di coloro che partecipano del regno terreno: i "risorti", non meglio specificati, gli "stranieri”, al loro servizio, che potranno anch'essi mangiare e bere, i peccatori, sofferenti e confusi per la vergogna. Inoltre, caratteristica della posizione di questi millenaristi è il ritorno delle nozze e della generazione, già criticato da Origene in precedenza, come abbiamo visto. Per Ireneo, invece, la condizione dei risorti nel regno è simile a quella angelica e non comporta la generazione di figli. Questa, in realtà, non è del tutto esclusa dal regno millenario, poiché è ancora propria di quei credenti -sia ex circumcisione sia ex gentibus - che, trovati in vita al momento del ritorno di Cristo, saranno governati dai santi e serviranno in Gerusalemme (V, 35, 1); si tratta, tuttavia, nella logica d'Ireneo (cf. $A d v$. haer. IV, 38, 3) di una generazione non più governata dalla concupiscenza, ma anch'essa dallo Spirito ${ }^{36}$, e comunque limitata ai superstiti al momento del ritorno di Cristo.

Insomma: ferme restando le differenze "strutturali" nella mentalità dei due autori (la carne e il mondo materiale voluti come beni da Dio nel suo unico progetto creativo, secondo Ireneo, conseguenza del peccato delle creature razionali, secondo Origene), il confronto dei testi mi sembra metta in luce sia una forte distanza della posizione d'Ireneo da quella del millenarismo materialista degli avversari di Origene, che appaiono praticare una vera e propria esegesi giudeo-cristiana della Scrittura, sia anche alcune analogie tra la concezione del regno dei giusti d'Ireneo e quella del paradiso che Origene, a partire da II, 11, 3, comincia a descrivere.

36 Cf. A. Orbe, Teología de San Ireneo, III, 504-507. 


\section{Dossier scritturistico}

Ireneo, Adv. haer. V, 32-35

Gen

$$
13,14-15
$$

13,17

15,18

Sal 103, 30

Is $\quad 6,11-12$

$11,6-9$

13,9

26,19

$30,25-26$

$31,9-32,1$

54, 11-14

58,14

65, 18-22

65,25

Ger
Bar

$4,36-5,9$

Ez

$37,12-14$

$28,25-26$

Dn

7, 27

12,13

Mt $\quad 5,5$

26, 27-29

Lc

12, 37-38

detti del Signore tramite $i$ presbiteri e Papia

Rm

8, 19-21

Gal

4,28

$23,7-8$

$31,10-14$
3,16

Ap $\quad 20,6$ millenaristi di Princ. II, 11, 2

Is $\quad 54,11-12$

60, 5-6. 10-11

$61,5-6$

65, $13-16$

Mt 26, 29

5,6

Lc 19, 19

Ap 21, 10-11.18-20

Afferma, com'è noto, il maestro di Lione: "allora regneranno sulla terra i giusti, crescendo grazie alla manifestazione del Signore e, per mezzo di lui, si abitueranno ad accogliere la gloria del Padre e accoglieranno, nel regno, la convivenza con i santi angeli e la comunione e l'unione delle cose spirituali" (V, 35, 1). "Così [l'uomo] veramente si eserciterà all'incorruttibilità, crescerà e giungerà alla pienezza dei tempi del regno, per divenire capace di accogliere la gloria del Padre" (V, 35, 2).

Quanto a Origene, in questa sezione del De principiis egli, contrariamente al solito, colloca il paradiso sulla terra ${ }^{38}$. In esso i beati si nutrono di verità e sapienza, conoscono il significato delle realtà terrestri, il significato d'Israele e delle sue leggi, l'azione della provvidenza (II, 11, 4-5).

L'utilizzo di $A p 21$ (la nuova Gerusalemme dal cielo) mi sembra un indizio molto forte della distanza ideologica tra i due millenarismi: Ireneo (Adv. Haer. V, 35, 2) la cita in riferimento alla "città di Dio" successiva al Millennio, vale a dire alla beatitudine definitiva di coloro che, passata la scena di questo mondo, avranno accesso alla gloria di Dio; invece i millenaristi criticati da Origene appaiono utilizzarla per descrivere la ricostruita Gerusalemme terrena, ove hanno luogo i godimenti millenari.

38 Se non è un fraintendimento di Rufino: cf. A. Monaci, «Cosmo», in: Ead. (dir.), Origene. Dizionario, 92-98, spec. 94. 
Si compie, dunque, in tale condizione, una prima fase della progressiva realizzazione della conoscenza che, proseguendo nei cieli, avvicina sempre di più l'anima al suo creatore (II, 11, 6). Commenta in conclusione Origene: "Così la natura razionale cresce gradatamente: non come cresceva in questa vita in anima e corpo (o carne), ma perché la mente, potenziata nell'intelligenza e nella sensibilità e giunta alla perfezione, raggiunge la conoscenza perfetta, non più impedita dai sensi della carne ma incrementata dai sensi dell'intelletto, osservando chiaramente e, per così dire, faccia a faccia $[1 \operatorname{Cor} 13,12]$ le cause delle cose" (II, 11, 7).

Non è difficile notare l'analogia nella funzione pedagogica e propedeutica tra il paradiso origeniano del De principiis e il regno dei giusti d'Ireneo, così come la loro comune lontananza da concezioni rozzamente materialistiche della vita dei risorti. 
Riassunto: L'articolo analizza specialmente De principiis II, 10,1-3; 11,1-7. Le caratteristiche della concezione origeniana della resurrezione dei morti (universalità, trasformazione del sostrato materiale, fine delle passioni, condizione intermedia e pedagogica) e la centralità di 1 Corinzi 15 nella riflessione dell'Alessandrino sono messe a confronto con alcune fonti anteriori (tra le quali 3Corinzi, Pseudo-Giustino, Ireneo, Tertulliano), per rilevare differenze e punti di contatto ideologici ed esegetici. Alcune analogie tra il regno dei giusti d'Ireneo e il paradiso di Origene sono particolarmente evidenti, nonostante le differenti impostazioni ideologiche dei due autori.

Parole chiavi: Resurrezione dei morti; esegesi di 1Corinzi 15; Ireneo, Adv.Haer. V,32-35; Millennio; paradiso.

Abstract: The paper especially examines Deprincipiis II, 10,1-3; 11,1-7. The features of Origen's idea of resurrection of the dead (universality, transformation of the material substratum, end of the passions, intermediate and pedagogic condition) and the great importance of 1Corinthians 15 in the Alexandrian's reflection are compared with some earlier sources (among which 3Corinthians, Pseudo-Justin, Irenaeus, Tertullian), to highlight ideological and exegetical differences and points of contact. Some analogies between Irenaeus' kingdom of just and Origen's paradise are particularly evident, despite the different ideological frameworks of the two authors.

Keywords: Resurrection of the dead, exegesis of 1 Corinthians 15; Irenaeus $A d v$. Haer. V,32-35; Millennium; paradise. 